\title{
Wavelength and intensity dependent primary photochemistry of isolated Photosystem II reaction centers at $5^{\circ} \mathrm{C}$
}

\author{
Scott R. Greenfield ${ }^{a}$, Michael Seibert ${ }^{b}$, Govindjee ${ }^{c}$, \\ Michael R. Wasielewski ${ }^{\text {a,d,* }}$ \\ a Chemistry Division, Argonne National Laboratory, Argonne, IL 60439-4831, USA \\ b Basic Sciences Center, National Renewable Energy Laboratory, Golden, CO 80401, USA \\ ${ }^{c}$ Department of Plant Biology, University of Illinois, Urbana, IL 61801-3707, USA \\ ${ }^{d}$ Department of Chemistry, Northwestern University, Evanston, IL 60208-3113, USA
}

Received 29 December 1995

\begin{abstract}
The long wavelength absorption band of the isolated Photosystem II reaction center was directly excited at five wavelengths between 655 and $689 \mathrm{~nm}$ to study the effects of excitation wavelength and intensity on both excitation energy transfer and charge separation processes. Subpicosecond transient absorption measurements were made monitoring principally the bleach of the pheophytin $a \mathrm{Q}_{x}$ band at $544 \mathrm{~nm}$. At all pump wavelengths, the kinetics require three exponentials $(1-3,10-25$ and $50-100 \mathrm{ps})$ to be fit properly. The pump energy was varied by a factor of twenty-five (40-1000 nJ), with no apparent effect on either the rates or the amplitude ratios of the three components, although clear evidence of nonlinear behavior was observed at the higher excitation energies. The dependence of both the rates and amplitude ratios of the three components upon pump wavelength will be discussed in terms of excitation energy transfer occurring on a 30 ps timescale. Selective excitation into the short and long-wavelength sides of the composite $Q_{y}$ band give identical transient spectra at 500 ps, indicating near-unity efficiency of excitation energy transfer. At $1 \mathrm{ps}$, the spectra are quite different, calling into question the extent of ultrafast ( $\sim 100 \mathrm{fs}$ ) excitation energy transfer. The time after the excitation pulse at which the transient crosses $\Delta A=0$ was found to be a highly sensitive measure of both the excitation energy and the identity of the pigment pool that had been excited.
\end{abstract}

\section{Introduction}

One of the two primary charge separation events in oxygenic photosynthesis occurs in the reaction center (RC) of photosystem II (PSII) [1]. The PSII $\mathrm{RC}$, the $\mathrm{D}_{1}-\mathrm{D}_{2}$-cytochrome $b$-559 complex, was first isolated by Nanba and Satoh in 1987 [2] (see review, [3]) and understanding its excitation energy

\footnotetext{
* Corresponding author.
}

transfer and charge separation processes has been an area of great interest ever since. The original isolation procedure for the PSII RC complex resulted in a preparation containing four-to-five chlorophyll $a(\mathrm{Chl}$ a) per RC. Later, more stable preparations were produced by substituting dodecyl $\beta$-maltoside for Triton X-100 [4], resulting in a stoichiometry of six Chl $a$, two pheophytin $a$ (Pheo $a$ ), two $\beta$-carotene, and one cytochrome $b-559$ heme per RC [1]. The lowest excited singlet states of the eight 
chlorophyll-like pigments all absorb within the RC composite $\mathrm{Q}_{y}$ band; the peak wavelength of the combined bands is at about $676 \mathrm{~nm}$ at room temperature for a sample immediately after removal from the isolation column. The primary electron donor, P680, is believed to peak at $680-682 \mathrm{~nm}[5,6]$ and is considered to be either a Chl $a$ dimer $[7,8]$ (analogous to the bacterial special pair) or a multimer of several Chl $a[9,10]$. Of the two Pheo $a$, only one is active (again, analogous to the bacterial system), with the peak of the active one at $\sim 682 \mathrm{~nm}$ (almost isoenergetic with P680) $[11,12]$ and the peak of the inactive one at $\sim 672 \mathrm{~nm}[12,13]$. The accessory Chl $a$ molecules which are not part of P680 (four, if P680 is a dimer) are also believed to peak at $\sim 670$ $\mathrm{nm}$ (see Ref. [1]), although it has been suggested that at least one Chl $a$ not associated with P680 absorbs near $680 \mathrm{~nm}$ [14]. Excitation energy transfer links the pigments on the blue side of the composite $\mathrm{Q}_{y}$ band (the "blue" pool) with the active redox pair on the red side of this absorption band (the "red" pool). It has been suggested that the "blue" pool itself is divided into two groups, one of which undergoes rapid (subpicosecond) excitation energy transfer to the "red" pool [15], and the other, weakly coupled to P680, undergoing excitation energy transfer on a tens of picoseconds timescale [16-18]. Only at low temperatures are the two main pools directly observable as separate peaks [9]. This tremendous spectral congestion greatly complicates efforts to understand the PSII RC and is in sharp contrast to the well-resolved absorption bands of the chromophores of the bacterial RC (see reviews, $[19,20]$ ).

While the fate of the charge-separated state in the isolated PSII RC has been well established (recombination to yield the excited singlet or the triplet state) [1], the nature of the excitation energy transfer and charge separation processes has been widely debated (see review [21]). Near room temperature, the RC kinetics have been studied by both time resolved Chl $a$ fluorescence [22-24] and transient absorption techniques [15-18,25-33]. While the time-resolved fluorescence measurements are hindered by the presence of processes occurring faster than the time resolution of the instrument, the transient absorption measurements are hampered by the lack of unambiguous indicators differentiating excitation energy transfer from charge separation. Never- theless, there are a number of features of transient absorption spectra that have been used to attempt to decipher the kinetics. Much of the work has been done probing the composite $\mathrm{Q}_{\text {y }}$ band itself [1517,25-28,31-34]. Because of the spectral congestion of the six Chl $a$ and two Pheo $a$ pigments in this band, it is difficult to use bleaching as an indicator of ground state depopulation of a specific pigment or group thereof. Furthermore, the small Stokes shift of the fluorescence (3-4 nm) [6] causes the main stimulated emission band to be superimposed upon the bleach, further complicating the interpretation of the kinetics. On the other hand, the $\mathrm{Q}_{y}(1,0)$ stimulated emission sideband at $\sim 740 \mathrm{~nm}$ has been used as a monitor of the population of the fluorescing state free from the effects of the $Q_{y}$ bleach $[29,32,33]$. The loss of Pheo a ground state population due to both excited singlet state formation (i.e., ${ }^{1 *}$ Pheo a) and charge separation can be monitored at the Pheo $a \mathrm{Q}_{x}$ band at $\sim 544 \mathrm{~nm}[18,28,30-33]$. While the data from this feature are complicated by the presence of bleaching due to ${ }^{1 *}$ Pheo $a$ (from both direct excitation of and excitation energy transfer to Pheo $a$ ), it is still probably the best indicator of charge separation that has been found so far.

Virtually all of the time-resolved experiments on PSII RCs near room temperature have detected both a $\sim 3$ and a $\sim 20$ ps component in the kinetics. The controversy as to which component is related to excitation energy transfer and which is related to charge separation is as much about the interpretation of the data as the data itself. Experiments at cryogenic temperatures, however, are all in agreement that $1-2$ ps is the measured time for charge separation $[6,34,35]$, and $80-100 \mathrm{ps}$ is the time for excitation energy transfer-limited charge separation (at 77 K) [34]. While several experiments with subpicosecond time resolution have been performed near room temperature that selectively excited each of the two pigment pools $[15,16,29,36]$, the extremely broad bandwidth of the "red"'-pool excitation pulse $(\sim 17$ $\mathrm{nm})$ [15] forced an excitation wavelength of $694 \mathrm{~nm}$ (i.e., far out into the wing of the band) to achieve any selectivity, thus precluding the use of more than one wavelength for excitation of the "red" pool. We have undertaken a new transient absorption study that utilizes an optical parametric amplifier (OPA) as a pump source. Wavelength tuning with the OPA can 
be achieved in a few minutes, allowing the same sample to be utilized for multiple excitation wavelengths. This device provides sub-200 fs time resolution with near transform-limited spectral bandwidths [37]. These narrow bandwidths ( $\sim 6 \mathrm{~nm})$ allow us to investigate the pump-wavelength dependence of the kinetics across the composite $\mathrm{Q}_{y}$ band. Experiments comparing selective excitation of the two pigment pools have the potential to provide insight into the identification of excitation energy transfer and charge separation processes, as selective excitation of the "blue" pool necessitates the occurrence of energy transfer, while selective excitation of the "red" pool minimizes the occurrence of inter-pool energy transfer. Thus, differences in the "blue"- versus "red"pool kinetics can be ascribed to energy transfer from the "blue" pool to the "red" pool.

Transient absorption kinetics were measured at the peak of the Pheo $a$ bleach, and transient spectra were taken over the 500 to $610 \mathrm{~nm}$ wavelength region. At 1 ps, excitation into the "blue" pigment pool of the composite $\mathrm{Q}_{y}$ band results in a significantly different transient spectrum than excitation into the "red" pool. The difference in the two spectra is shown to be very similar to the transient spectrum of ${ }^{1 *} \mathrm{Chl} a$ in vitro. At long time $(500 \mathrm{ps})$, the spectra and hence charge separation yields are indistinguishable, indicating near-unity quantum yield for excitation energy transfer from the "blue" pool to the "red" pool. The kinetics of the Pheo $a$ bleach are triple exponential at all excitation wavelengths used. Finally, the time at which the decay crosses $\Delta A=0$ is shown to be a powerful indicator of pump intensity and the identity of the pigment pool that had been excited [18].

\section{Materials and methods}

$\mathrm{D}_{1}-\mathrm{D}_{2}$-cytochrome $b$-559 PSII RC complex was isolated from PSII-enriched appressed membrane fragments obtained from market spinach [4]. PSII membranes (120 mg Chl a) were pelleted; resuspended in $50 \mathrm{mM}$ Tris- $\mathrm{HCl}(\mathrm{pH} \mathrm{7.2)}, 30 \mathrm{mM} \mathrm{NaCl}$, and $4 \%$ Triton $\mathrm{X}-100$ at $1 \mathrm{mg} \mathrm{Chl} a / \mathrm{ml}$, and incubated with stirring for $1 \mathrm{~h}$. All manipulations in this procedure were done at $4^{\circ} \mathrm{C}$ in the dark. The solubilized material was then centrifuged for $1 \mathrm{~h}$ at
$32000 \times g$, and the supernatant was loaded onto a buffer-equilibrated $1.6 \times 20 \mathrm{~cm}$ Fractogel TSKDEAE 650S (Supelco) column. The column was then washed with $50 \mathrm{mM}$ Tris- $\mathrm{HCl}(\mathrm{pH} 7.2), 30 \mathrm{mM}$ $\mathrm{NaCl}$, and $0.05 \%$ Triton buffer using an FPLC system (Pharmacia) until the absorption of the eluent at $670 \mathrm{~nm}$ was about $0.03 \mathrm{~cm}^{-1}$ (the spectrum of the eluent is like that of an isolated RC except that the red absorption peak was at $670 \mathrm{~nm}$ ). At that point, the column was washed with $150 \mathrm{ml}$ of $50 \mathrm{mM}$ Tris- $\mathrm{HCl}(\mathrm{pH} 7.2), 30 \mathrm{mM} \mathrm{NaCl}$, and $0.03 \% \mathrm{n}$ dodecyl $\beta$-D-maltoside (DM) to remove Triton. The RCs were then eluted with a $30-200 \mathrm{mM} \mathrm{NaCl}$ linear gradient in the same buffer. Green fractions obtained at around $125 \mathrm{mM} \mathrm{NaCl}$ were pooled, desalted and concentrated within $1 \mathrm{~h}$ using a Centriprep 50 Concentrator (Amicon), and stored at $-80^{\circ} \mathrm{C}$ until use. These RCs contain about $6 \mathrm{Chl} a$, $2 \beta$-carotenes, and 1 cytochrome $b-559$ per 2 Pheo $a$.

Experiments were performed on $\mathrm{Chl} a$ in tetrahydrofuran (THF) solution at room temperature. Chl $a$ was isolated from spinach [38]. The sample was purified on a TLC plate in the dark immediately prior to preparation of the solution. The peak of the $\mathrm{Q}_{y}$ band was at $664 \mathrm{~nm}$, with an absorbance of 0.62 . A peak extinction coefficient of 79000 [39] was used for calculation of the sample concentration $(7.8 \times$ $\left.10^{-6} \mathrm{M}\right)$. The THF was distilled from lithium aluminum hydride and stored over molecular sieves under $\mathrm{N}_{2}$.

A sample temperature of $\sim 5^{\circ} \mathrm{C}$ for the PSII RCs was maintained by flowing ice-water through the cooling jacket of a $1 \mathrm{~cm}$ path length fused silica spectroscopic cell (Starna). The rate of sample degradation is greatly decreased by keeping the sample at this temperature (as compared to room temperature). No significant changes in either the composite $Q_{y}$ band peak position or the second derivative of the band were discernible after at least an hour of illumination. The sample was continuously stirred; this avoided accumulation of RCs in the triplet state in the sample volume interrogated by the laser beam. Steady-state spectral measurements were done with a Shimadzu UV160 spectrometer.

Transient absorption measurements were performed with an optical parametric amplifier [37] (OPA) pumped by the second harmonic of an ampli- 
fied $\mathrm{Ti}$ : sapphire laser system operating at $1.3 \mathrm{kHz}$. The pulse length of the pump pulses was $\sim 180 \mathrm{fs}$, and bandwidths (FWHM) of $\sim 6 \mathrm{~nm}$ (near transform-limited) were typical. A white light continuum was generated from the amplified fundamental in a fused silica block, and was split into a probe beam and a reference beam. The polarization purity of both the pump and probe beams was very high, as both beams pass through calcite polarizers before reaching the sample. Like all of the previous work on PSII RCs done in our laboratory $[18,25,31,35]$, the probe beam was polarized at the magic angle $\left(54.7^{\circ}\right)$ with respect to the pump beam. This polarization configuration, unlike having the two beams with parallel or perpendicular polarizations, allows direct observation of population kinetics without artifacts due to polarization phenomena. The importance of measuring the isotropic kinetics (either by direct measurement with the magic angle configuration or by calculation from both parallel and perpendicular data) should be stressed, as data on PSII RCs using the parallel polarization configuration continue to be presented [16,36].

Pump and probe beams were crossed in the sample with a spot size (diameter) of $\sim 250 \mu \mathrm{m}$. Pump energies were varied from $40 \mathrm{~nJ}$ to $1.0 \mu \mathrm{J}$. At 100 $\mathrm{nJ}$, the energy density of the pump (the parameter of interest when comparing to other work) was $\sim 200$ $\mu \mathrm{J} / \mathrm{cm}^{2}$. Both the probe and reference beams were focused into a monochromator with both the entrance and exit slits set at a $4 \mathrm{~nm}$ bandwidth. After exiting the monochromator, the intensities of the two beams were measured by separate $\mathrm{Si}$ photodiodes. The photodiode output was captured by gated integrators and digitized by a 12-bit A/D board. Every other pump pulse was blocked by a mechanical chopper, and a $\Delta A$ value was calculated for each pair of pulses. Kinetic scans were typically the average of 10-20 delayline passes, with each point of each pass being the average of 100 laser shots. The scans were broken down into three temporal regions, with 100 fs per point from -3 to $+17 \mathrm{ps,} 1$ ps per point from 17 to $122 \mathrm{ps}$, and 5 ps per point from 122 to 600 ps. Each data set was individually fit to a triple exponential decay and a nondecaying component using a grid search routine. Transient spectra were measured by setting the time between the pump and the probe pulses at a fixed delay and scanning

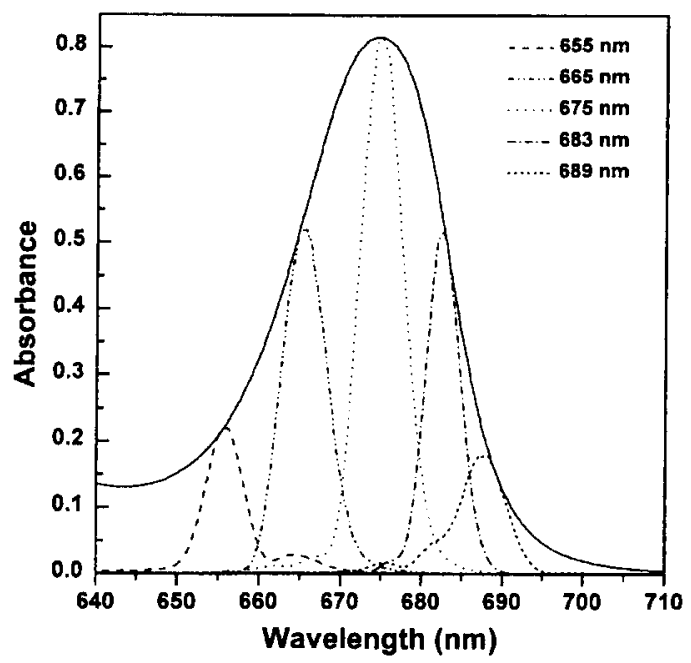

Fig. 1. Steady-state absorption spectrum of the composite $\mathrm{Q}_{y}$ band of isolated PSII RCs in a $1 \mathrm{~cm}$ cell. Superimposed are the excitation profiles (product of the pump and RC spectra - see text for details) for the five pump wavelengths. The actual spectral bandwidths for the pump pulses (not shown) are $\sim 6 \mathrm{~nm}$ (neartransform-limited).

the wavelength of the monochromator $(0.75 \mathrm{~nm}$ per point). Thus the spectra presented are direct spectra, not ones calculated from the results of global analysis fits of kinetic scans at numerous wavelengths.

Pump wavelengths of $655,665,675,683$, and 689 $\mathrm{nm}$ were used. The effect of the finite bandwidth ( $\sim 6 \mathrm{~nm}$ ) of the pump pulses on the absorption of the RCs can be calculated by taking the product of the normalized spectral intensity profile of the pump pulse and the absorption spectrum of the RC. Fig. 1 shows these "excitation profiles" for the five pump wavelengths superimposed upon the spectrum of the composite $\mathrm{Q}_{y}$ band of the RC. Selective excitation of the "blue" side of the $\mathrm{Q}_{y}$ band can be achieved with 655 and $665 \mathrm{~nm}$ pump wavelengths, whereas 683 and $689 \mathrm{~nm}$ pump wavelengths result in selective excitation of the "red" side of the band. A pump wavelength of $675 \mathrm{~nm}$ excites the peak of the band, and no selectivity is achieved. It should be noted that the spectral intensity profile of the pump is similar for all wavelengths, and that the structure of the excitation profiles for 655 and $689 \mathrm{~nm}$ in Fig. 1 is due to the much higher sample extinction coefficient at the center of the $Q_{y}$, band. For all five pump wavelengths, the peak of the excitation profile is within $1 \mathrm{~nm}$ of the pump wavelength. 
The five pump wavelengths chosen correspond roughly to the peak, half-maxima and quarter maxima wavelengths for the composite $\mathrm{Q}_{y}$ band absorption. The true optical density of the sample for the actual laser pump pulses can be determined by integrating the excitation profile over wavelength and dividing by the integrated pump spectral intensity profile:

$\mathrm{OD}_{\text {effective }}=\frac{\int I_{\text {pump }}(\lambda) \mathrm{OD}_{\text {sample }}(\lambda) \mathrm{d} \lambda}{\int I_{\text {pump }}(\lambda) \mathrm{d} \lambda}$,

where $I_{\text {pump }}$ is the normalized pump spectral intensity profile, $\operatorname{OD}_{\text {sample }}(\lambda)$ is the optical density of the sample as a function of pump wavelength, and $\mathrm{OD}_{\text {effective }}$ is the true optical density of the sample for the finite bandwidth laser pulses. Sample concentrations were adjusted to keep the OD near 0.5 at the excitation wavelength, with relative concentrations of $4: 2: 1: 2: 4$ for $655,665,675,683$ and $689 \mathrm{~nm}$, respectively; the calculated optical densities (using Eq. 1) are $0.47,0.53,0.38,0.47$, and 0.38 . For the 665 and $683 \mathrm{~nm}$ pump wavelengths, the OD at the peak of the $Q_{y}$ band was $\sim 0.8$, which corresponds to a $\mathrm{RC}$ concentration of $\sim 2 \times 10^{-6} \mathrm{M}$.

Some discussion at this point is warranted about the various ways to display the intensity dependence of observables over the different pump wavelengths. The transient absorption signal is directly proportional to the number of photons absorbed by the sample. For a given pump energy, this quantity changes as a function of pump wavelength due to two factors: the optical density of the sample (as discussed above), and the change in the number of photons (a small effect - about 5\% between 655 and $689 \mathrm{~nm}$ ). Much of the intensity-dependent data discussed below will compare excitation wavelengths of 665 and $683 \mathrm{~nm}$. As the correction for absorbed photons between these two wavelengths is only $3.5 \%$, the correction will be ignored and the data will be displayed simply as a function of incident pump energy. Because the correction is significantly higher (as much as $20 \%$ ) between the other pump wavelengths, that data will be presented in terms of absorbed photons per $\mathrm{cm}^{2}$. We will also use an alternative way to display the data which clearly show the effects of multiple excitation of RCs at high pump energies (vide infra), where the data will be displayed in terms of absorbed photons per RC. It should be noted that the experiments on Chl $a$ in THF will show that, at high pump energies, the absorption of the sample becomes sublinear. No correction for this phenomena is made in any of the figures: the number of photons absorbed is always calculated assuming linear absorption.

\section{Results}

\subsection{Kinetics at $544 \mathrm{~nm}$ with $200 \mathrm{~nJ}$ pump pulses}

Transient absorption kinetics probing at $544 \mathrm{~nm}$ are shown in Fig. 2. The pump energy is $200 \mathrm{~nJ}$ and the wavelength is $683 \mathrm{~nm}$. The data are fit to a triple exponential decay (two exponentials did not produce satisfactory residuals) and a component that does not decay on the sub-nanosecond timescale of the experiment. Typical lifetimes of the triple exponential decay with the $683 \mathrm{~nm}$ pump are 1-2,10-15 and 60-70 ps. The nondecaying component (hereafter referred to as the shelf) presumably reflects the fully

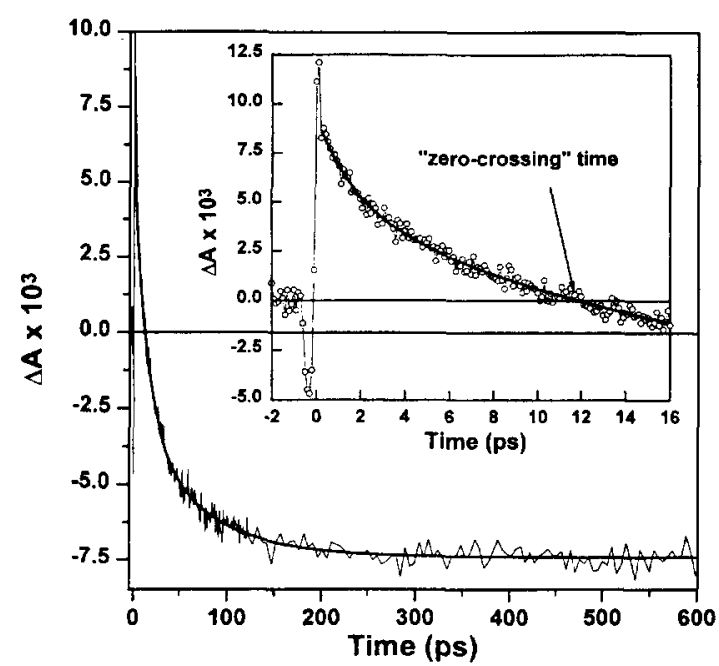

Fig. 2. Transient absorption kinetics probing at $544 \mathrm{~nm}$ in isolated PSII RCs. The pump energy and wavelength are $200 \mathrm{~nJ}$ and 683 $\mathrm{nm}$, respectively. Inset shows the same data on a shorter timescale. The triple exponential fit to the data is shown in both. The large oscillations near $t=0$ are due to the buffer/cell system, and are unrelated to the PSII RC dynamics. Note the "zero-crossing" time of $\sim 11.5 \mathrm{ps}$. 
Table 1

Averages and standard deviations for the fit parameters to the kinetic data at $544 \mathrm{~nm}$ using a pump energy of $\sim 200 \mathrm{~nJ}$

\begin{tabular}{|c|c|c|c|c|c|c|c|c|c|c|}
\hline$\lambda_{\text {pump }}$ & $\begin{array}{l}\tau_{\text {fast }} \\
\text { (ps) }\end{array}$ & $A_{\text {fast }}$ & $\% A_{\text {fast }}$ & $\begin{array}{l}\tau_{\text {int }} \\
\text { (ps) }\end{array}$ & $A_{\text {int }}$ & $\% A_{\text {int }}$ & $\begin{array}{l}\tau_{\text {slow }} \\
\text { (ps) }\end{array}$ & $A_{\text {slow }}$ & $\% A_{\text {slow }}$ & Shelf \\
\hline 665 & $2.7 \pm 0.4$ & $\begin{array}{l}0.0044 \\
\pm 0.0005\end{array}$ & $21 \pm 2$ & $22 \pm 2$ & $\begin{array}{l}0.0125 \\
\pm 0.0012\end{array}$ & $60 \pm 3$ & $98 \pm 16$ & $\begin{array}{l}0.0039 \\
\pm 0.0008\end{array}$ & $19 \pm 4$ & $\begin{array}{l}-0.0069 \\
\pm 0.0005\end{array}$ \\
\hline 683 & $1.6 \pm 0.4$ & $\begin{array}{l}0.0034 \\
\pm 0.0006\end{array}$ & $20 \pm 3$ & $13 \pm 2$ & $\begin{array}{l}0.0077 \\
\pm 0.0008\end{array}$ & $46 \pm 2$ & $64 \pm 5$ & $\begin{array}{l}0.0057 \\
\pm 0.0009\end{array}$ & $34 \pm 4$ & $\begin{array}{r}-0.0076 \\
\pm 0.0010\end{array}$ \\
\hline
\end{tabular}

The data are fit to a triple exponential decay and a nondecaying component (see text for details).

The amplitudes of the three decay components are given as both absolute amplitudes and as a percentage of the total decay amplitude.

charge separated state of the $\mathrm{RC}\left(\mathrm{P} 680^{+}-\mathrm{Pheo} a^{-}\right)$, which has been shown by nanosecond experiments to have a lifetime of $36 \mathrm{~ns}$ at ambient temperature [40]. The difficulties in reliably fitting a triple exponential decay with the modest signal-to-noise (typically about 50 at this pump energy) inherent in transient absorption experiments is well known. It was thus imperative to scan the data until they reached a "flat" (on the timescale in question) baseline, and to record at least several factors of " $e$ " in the decay of the slowest component. This was especially important given that the relative amplitude of the slow component was $20-30 \%$ (see below) in our case; i.e., it is weak enough to be difficult to fit, yet strong enough to cause a nontrivial effect on the other components if it is not fit properly. When we fit our data out to only $70 \mathrm{ps}$, it is well fit to a biexponential with 2 and 21 ps lifetimes. These lifetimes closely match those of Klug et al. [32], whose data extended to only $70 \mathrm{ps}$ in spite of the fact that the transient was still clearly decaying at that time.

The inset to Fig. 2 shows the same data up to 16 ps only. The ultrafast oscillations around $t=0$ are due to an interaction of the pump and probe pulses with the buffer/cell system, and have been observed before by Hong et al. [41]. This signal is roughly linear with pump energy, and as such it cannot be removed by going to lower pump energies. The data are fit only after the oscillations have completely damped out, which is $300-400 \mathrm{fs}$ after the peak of the positive transient. Also shown in the inset is the "zero-crossing" time (see below), which is simply the time after the excitation pulse at which the decay crosses $\Delta A=0$ while probing at $544 \mathrm{~nm}$.

The result of averages of the fits of multiple scans at pump wavelengths of 665 and $683 \mathrm{~nm}$ are given in
Table 1. For all scans used in this table, the pump energy was 200 to $220 \mathrm{~nJ}$, and the probe wavelength was $544 \mathrm{~nm}$. The average at both wavelengths is over six scans, with each scan having a "signal-tonoise" (S/N) ratio ranging from $46: 1$ to $84: 1$. The $\mathrm{S} / \mathrm{N}$ ratio is calculated by dividing the total decay amplitude (i.e., $\Delta A$ at $\sim 350 \mathrm{fs}$ minus $\Delta A$ at long time) by the RMS deviation of the fit. The $\mathrm{S} / \mathrm{N}$ at $665 \mathrm{~nm}$ is slightly better than at $683 \mathrm{~nm}$ simply because of the larger amplitude at $t=0$ when pumping into the blue side of the band (vide infra). The amplitudes of the components are given both as absolute amplitudes and as a percentage of the total decay amplitude.



Fig. 3. Absolute amplitudes of the three kinetic components to the triple exponential fit of the transient absorption decay at $544 \mathrm{~nm}$ as a function of pump energy with an excitation wavelength of $683 \mathrm{~nm}$ in isolated PSII RCs. The lines are linear fits to the data for pump energies up to $200 \mathrm{~nJ}$. 


\subsection{Intensity dependence of kinetics}

The amplitudes of the three kinetic components as a function of pump energy with an excitation wavelength of $683 \mathrm{~nm}$ and a probe wavelength of $544 \mathrm{~nm}$ are shown in Fig. 3. Error bars $( \pm 1 \sigma)$ are given, where applicable. All three components are linear with pump energy up to 200-250 nJ. Above this point, all three amplitudes continue to increase, but at a lesser slope. Similar results were obtained with a $665 \mathrm{~nm}$ pump wavelength (data not shown).

In order to determine whether the sublinear behavior at high pump energies as seen in Fig. 3 affects any of the three kinetic components differently, we
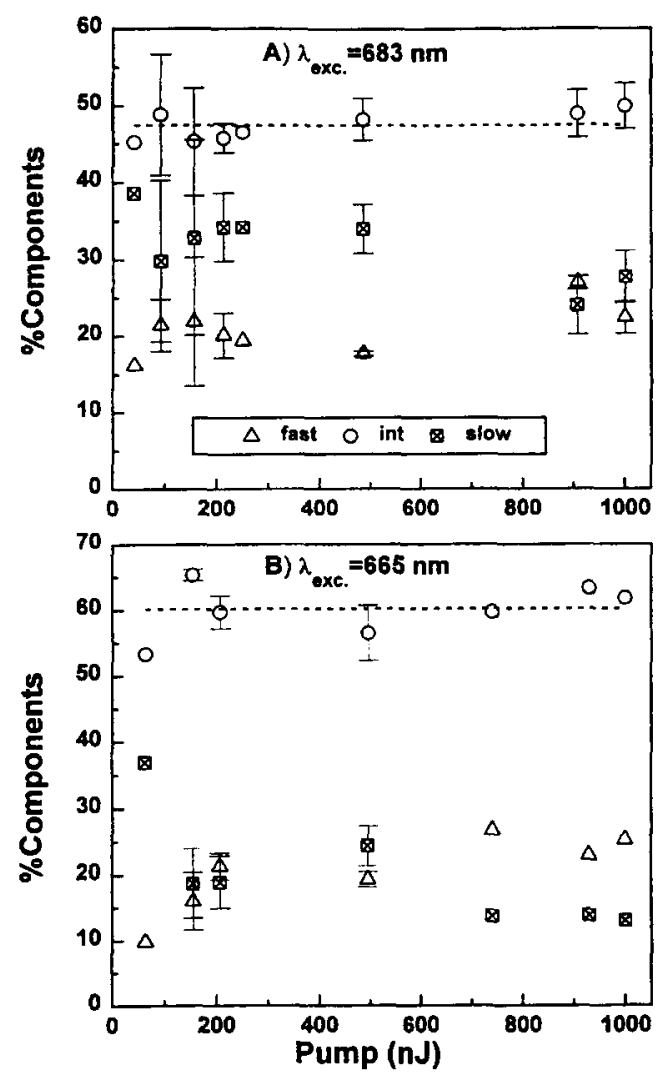

Fig. 4. Relative amplitudes of the three kinetic components to the transient absorption data probing at $544 \mathrm{~nm}$ in isolated PSII RCs as a function of pump energy. (A) $683 \mathrm{~nm}$ pump. (B) $665 \mathrm{~nm}$ pump. The dashed lines are the averages of the values for the intermediate component at each pump wavelength. Note the independence of the data with respect to pump energy, and the larger amplitude of the intermediate component with the $665 \mathrm{~nm}$ pump.
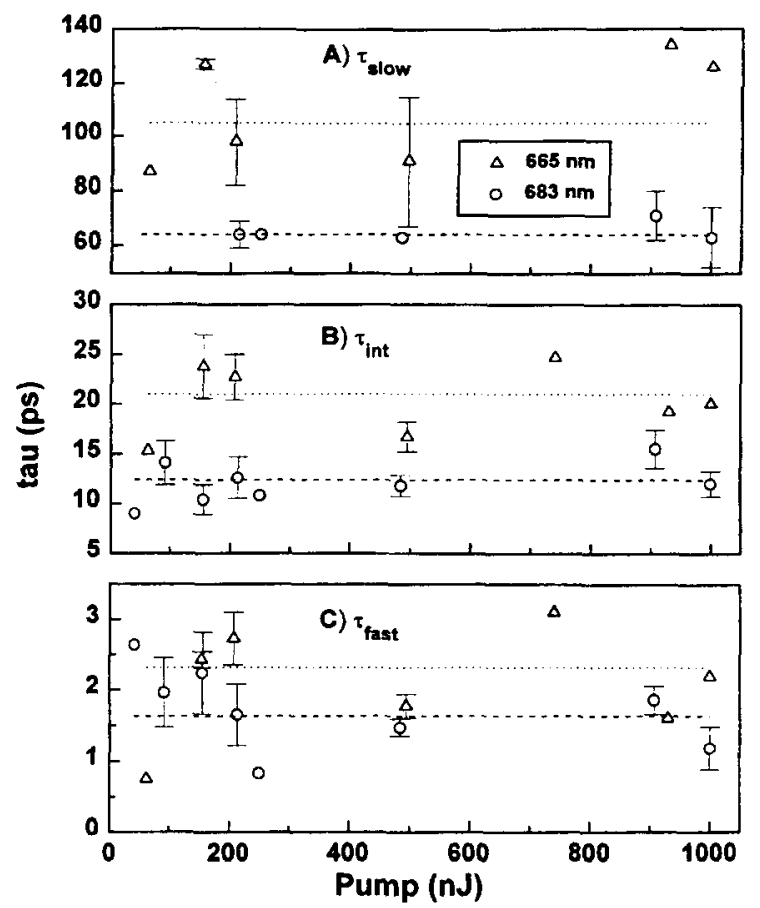

Fig. 5. Time constants for the triple exponential decay of the transient absorption signal at $544 \mathrm{~nm}$ in isolated PSII RCs. Data for pump wavelengths of 665 and $683 \mathrm{~nm}$ are shown. Dot and dashed lines are the averages over all pump intensities for 665 and $683 \mathrm{~nm}$, respectively. (A) slow component, $\tau_{\text {slow }}$; (B) intermediate component, $\tau_{\text {int }} ;(\mathrm{C})$ fast component, $\tau_{\text {fast }}$. For all three components, the $665 \mathrm{~nm}$ time constants are slower than the $683 \mathrm{~nm}$ ones. Note the absence of any intensity dependence of the time constants.

have plotted the amplitudes of the components on a percentage basis as a function of $683 \mathrm{~nm}$ pump energy in Fig. 4A. The largest component is the intermediate component, with $47 \pm 5 \%$ of the amplitude. This percentage is independent of the pump energy over the range investigated, and the dashed line is the mean of all of the values. The amplitude of the fast component is $\sim 20 \%$, and that of the slow component is $\sim 30-35 \%$. There is no statistically significant trend in the percentages of the fast and slow components up to $500 \mathrm{~nJ}$, and the difference at $0.9-1.0 \mu \mathrm{J}$ is fairly small.

Fig. 4B shows similar data, but for a pump wavelength of $665 \mathrm{~nm}$. Once again, the intermediate component is dominant $(60 \pm 4 \%)$, and its percentage is also independent of excitation energy. The 
amplitude percentage of the intermediate component when pumping at $665 \mathrm{~nm}$ is significantly higher than when pumping at $683 \mathrm{~nm}$, and will be discussed below in terms of excitation energy transfer. The fast and slow components vary from $15 \%$ to $25 \%$, with a change in one at the expense of the other. The trend toward the fast component becoming greater (and the slow component weaker) at pump energies above $700 \mathrm{~nJ}$ is probably not statistically significant, as there is only one scan per point in that region.

The dependence of the lifetimes on pump energy for 665 and $683 \mathrm{~nm}$ excitation pulses is shown in Fig. 5A-Fig. 5C. With $683 \mathrm{~nm}$ excitation, $\tau_{\text {slow }}$ and $\tau_{\text {int }}$ are both clearly independent of pump energy, with average time constants of $64 \pm 9$ and $12 \pm 2 \mathrm{ps}$, respectively. For $665 \mathrm{~nm}$ excitation, $\tau_{\text {slow }}$ and $\tau_{\text {int }}$ have average time constants of $105 \pm 21$ and $21 \pm 4$ ps, respectively, with no apparent dependence upon pump energy. One should note that, for $\tau_{\text {slow }}$, all of the $665 \mathrm{~nm}$ time constants are slower than the slowest one for $683 \mathrm{~nm}$ excitation. This same behavior is also seen for $\tau_{\text {int }}$ in Fig. 5B. Fig. 5C shows data for $\tau_{\text {fast }}$. While the scatter on these data make it difficult to prove the absence of any intensity dependence, none clearly stands out. The time constants for $\tau_{\text {fast }}$ with $665 \mathrm{~nm}$ excitation are generally slower than those for $683 \mathrm{~nm}$ excitation, with averages of $2.3 \pm$ 0.7 and $1.6 \pm 0.6 \mathrm{ps}$, respectively.

\subsection{Intensity dependence of $t=350$ fs amplitude}

Fig. 6 shows the amplitude of the transient absorption signal at $\sim 350 \mathrm{fs}$ after the excitation pulse for pump wavelengths of 665 and $683 \mathrm{~nm}$ as a function of pump energy. The amplitude at earlier time cannot be accurately determined, as the large oscillations from the buffer/cell system mask the behavior of the RCs at that time (see Fig. 2 inset). The amplitudes in this figure were derived from the fits to the data. As long as the data are well fit at early time (which it is), this method is preferable to picking the amplitude at a single arbitrary time delay, as the latter is susceptible to the inherently greater noise of a single point. For a given pump wavelength, this amplitude can be used as a measure of the total excited state population (at least in the linear regime). For both pump wavelengths, the amplitude is linear up to $200-250 \mathrm{~nJ}$, and then in-



Fig. 6. Amplitude of the transient absorption signal at $544 \mathrm{~nm}$ $\sim 350$ fs after the laser flash (i.e., the earliest time at which the data can be measured free from artifacts due to the buffer/cell response) as a function of pump energy for pump wavelengths of 665 and $683 \mathrm{~nm}$ in isolated PSII RCs. Linear fits to the data for pump energies up to $200 \mathrm{~nJ}$ are also shown.

creases at a lesser slope. Note that at all pump energies, the amplitude is greater for "blue"-pool excitation than for "red"'-pool excitation.

\subsection{Intensity dependence of Chl a in THF}

In order to gain insight into the cause of the deviation from linearity of the $t=350 \mathrm{fs}$ amplitude, we investigated the behavior of Chl $a$ in THF upon laser excitation. Although Pheo $a$ is also present in the composite $\mathrm{Q}_{y}$ band of the PSII RC, its spectral contribution is relatively minor compared to that of Chl $a$, which dominates because of both the relative pigment oscillator strengths and the stoichiometry. For this reason, only Chl $a$ was used for the in vitro experiments, two of which were performed. The first was to make the laser act like simple spectrophotometer: the laser intensity was measured before and after the sample, thus determining the percent transmitted energy, which of course allows a calculation of the absorption of the sample. This was done at many different pump energies from 10 to $850 \mathrm{~nJ}$. At the lowest pump energies, the measured optical density $(\sim 0.57)$ matches that measured by a UV-Vis spectrophotometer, taking into account the effect of 


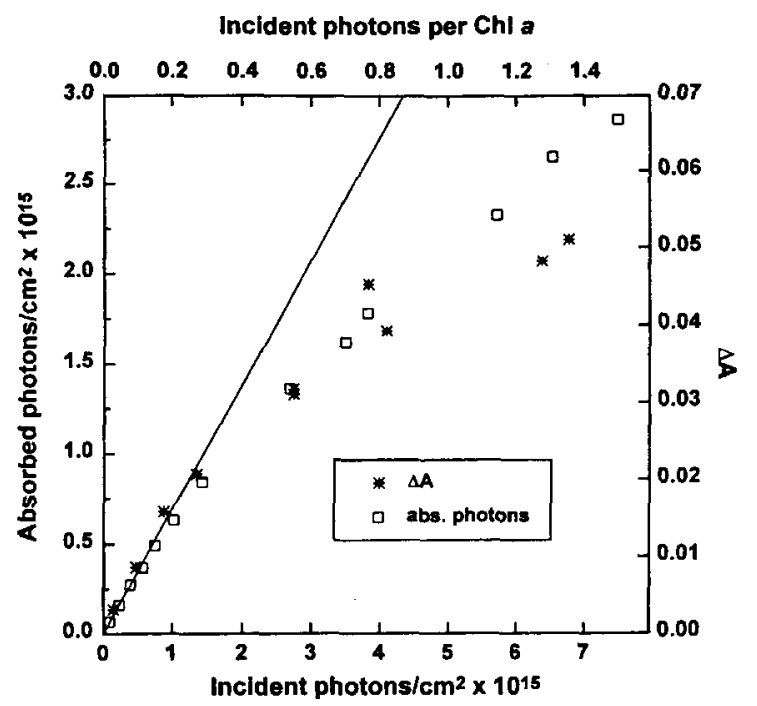

Fig. 7. Chl $a$ in THF experiments. Squares show the absorbed photons per $\mathrm{cm}^{2}$ as a function of incident photons per $\mathrm{cm}^{2}$. Deviation from linearity is due to populating a nontrivial fraction of the chromophores in the excited state during the pump pulse. The straight line ( $73 \%$ absorption) is determined from the spectrum of the sample in a UV-Vis spectrometer, adjusting for the effects of the finite bandwidth of the pump pulse. Asterisks show the transient absorption signal at early time probing at $544 \mathrm{~nm}$. Note that the deviation from linearity for the transient absorption data is virtually identical to the deviation of the absorbance measurement.

the finite bandwidth of the pump pulse as was discussed in Section 2 for the RCs. As the pump energy was increased, the optical density decreased, getting as low as $\sim 0.22$ at the highest pump energy used.

The second experiment was to measure the amplitude of the transient absorption signal at early time (the signal decays on a nanosecond timescale) at 544 $\mathrm{nm}$ for the Chl $a$ in THF solution as a function of pump energy. Fig. 7 shows these data, along with the percent transmission data described above reformulated in terms of absorbed photons $/ \mathrm{cm}^{2}$ versus incident photons $/ \mathrm{cm}^{2}$. As one can see, the two curves have essentially the same form. This should not be surprising, as the transient absorption signal is directly proportional to the number of absorbed photons. The straight line corresponds to $73 \%$ absorption (the low-energy limit). Below $1 \times 10^{15}$ incident photons $/ \mathrm{cm}^{2}$, both sets of data are well fit by the line. The sublinear behavior of the sample optical density (and therefore the transient absorption signal) is sim- ply a result of the pump intensity being high enough to go beyond the linear regime in which Beer's law holds. The deviation occurs because a nontrivial fraction of the sample is in its excited state at high pump intensities [42]. These excited pigments can interact with another photon in the pump pulse and result in stimulated emission, resulting in the "loss" of a previously excited pigment.

Is the sublinear behavior of the $t=350 \mathrm{fs}$ amplitude data in the isolated PSII RCs simply a measure of the same phenomena? The highest reasonably linear point for the Chl $a$ in THF experiments was at $\sim 0.29$ incident photons per molecule, which corresponds to 0.21 absorbed photons per molecule (assuming linear, i.e., $73 \%$, absorption). If we multiply this by 3.5 (for simplicity, assuming three Chl $a$ per pigment pool, plus one Pheo $a$ with half of the oscillator strength), this gives 0.74 absorbed photons per $R C$ for selective excitation of either pigment pool. This is roughly the upper limit of linearity (corresponding to $250 \mathrm{~nJ}$ for 665 and $683 \mathrm{~nm}$ ) for the $t=350 \mathrm{fs}$ data in Fig. 6. Since the point of deviation from linearity is quite consistent for the Chl $a$ in THF and the isolated PSII RC measurements, and the same sublinear behavior is observed in both samples, it seems quite reasonable to conclude that the behavior is due to the same phenomenon, i.e., a deviation from Beer's law linear absorption. Thus, the nonlinearity of the $t=350 \mathrm{fs}$ data for the isolated PSII RCs has nothing to do with the presence of multiple pigments within each RC (e.g., the possibility of annihilation phenomena). The sublinear behavior of the amplitudes of all three of the individual kinetic components (see Fig. 3) is presumably another manifestation of the Beer's law deviation, as the sublinearity begins at the same energy as with the $t=350 \mathrm{fs}$ data, and it affects all three components equally (see Fig. 4).

\subsection{Intensity dependence of the shelf}

With sufficiently high pulse energies, it is possible to excite multiple chromophores in the individual RCs. Of course, each RC is limited to a single charge separated state, as there is only one active redox pair per RC. Therefore, the saturation of charge separation is an excellent indicator of multiphoton 


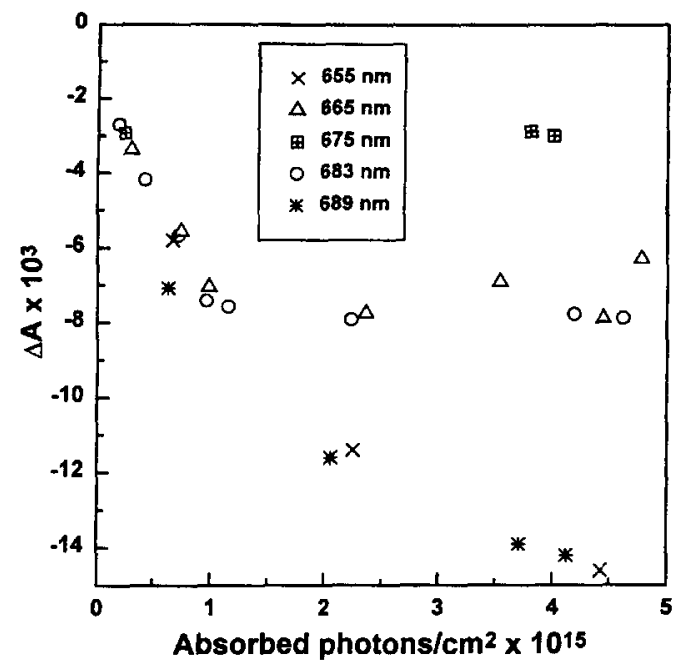

Fig. 8. Amplitude of the nondecaying component (shelf) at 544 $\mathrm{nm}$ as a function of absorbed photon density at different pump wavelengths in isolated PSII RCs. For pump wavelengths of 665 and $683 \mathrm{~nm}$, the data are linear up to $\sim 250 \mathrm{~nJ}$. Deviations from linearity are due to multiple excitation of individual RCs at high pump energies, and both the point of deviation from linearity and the saturation value of the shelf are dependent primarily on sample concentration, which is higher as the excitation wavelengths gets further from the composite $Q_{y}$ band peak (see text for details).

excitation of individual RCs, and the amplitude of the Pheo $a$ bleach is arguably the best indicator of the extent of charge separation occurring in the sample. The amplitude of the shelf of the transient absorption signal at $544 \mathrm{~nm}$ (equivalent to $\Delta A$ at $\sim 500$ ps; i.e., after all excitation energy and charge transfer is complete) is plotted in Fig. 8 as a function of absorbed photons per $\mathrm{cm}^{2}$ at all five pump wavelengths. The data for 665 and $683 \mathrm{~nm}$ are linear up to $\sim 250 \mathrm{~nJ}$, and then saturates at a slightly higher value for pump energies between 0.5 and $1.0 \mu \mathrm{J}$. The data for both wavelengths fall on the same curve, with the equivalence in the linear regime indicating that the quantum yield for charge separation is the same for both excitation wavelengths. This implies that the quantum yield for excitation energy transfer (i.e., for excitation at $665 \mathrm{~nm}$ ) is near unity. At 655 and $689 \mathrm{~nm}$ the composite $\mathrm{Q}_{y}$ band oscillator strength is roughly half of that at 665 and $683 \mathrm{~nm}$, and the sample concentration was therefore set to be double. As with the $665 / 683 \mathrm{~nm}$ data, the
$655 / 689 \mathrm{~nm}$ data lie on the same curve, thus indicating that the quantum yield for excitation energy transfer and charge separation is unchanged by going out further into the wings of the $Q_{y}$ band. At the highest pump intensity, the shelf for 655 and $689 \mathrm{~nm}$ excitation is twice that of the 665 and $683 \mathrm{~nm}$ data, as would be expected since twice as many RCs are excited at saturation at these wavelengths as are excited at 665 and $683 \mathrm{~nm}$. Similarly, the saturation level of the shelf for the $675 \mathrm{~nm}$ data is roughly half of that for the 665 and $683 \mathrm{~nm}$ data.

\subsection{Zero-crossing time}

The time at which the transient absorption decay for a probe wavelength of $544 \mathrm{~nm}$ crosses $\Delta A=0$ appears to be a useful monitor of a number of parameters, including the pump energy density, the sample activity, and the identity of the pigment pool that has been excited [18]. Fig. 9A shows the "zerocrossing" time with $655,665,683$, and $689 \mathrm{~nm}$ pump wavelengths for pump energies in the range of 40 to $1000 \mathrm{~nJ}$. The two lines are linear fits to the 665 and $683 \mathrm{~nm}$ data. The $655 \mathrm{~nm}$ data appear to fit a line of lesser slope but identical intercept as the 665 $\mathrm{nm}$ data. Likewise, the $689 \mathrm{~nm}$ data appear to have the same intercept as the $683 \mathrm{~nm}$ data, but also with a lesser slope. Thus, in the low intensity limit, the zero-crossing time is independent of wavelength within each of the pigment pools, but is quite different between the two pools. This implies that the zero-crossing time is related to pigment pool-specific phenomena.

The difference in slopes for the two wavelengths within each pigment pool is an indication of what is responsible for this phenomena. Since the slopes are different, it is clear that the zero-crossing time is not simply a function of the pump photon flux. Fig. 9B shows the same data plotted against absorbed photons per RC. Now the $689 \mathrm{~nm}$ data actually fall on the same line as the $683 \mathrm{~nm}$ data, and the $655 \mathrm{~nm}$ data fall on the $665 \mathrm{~nm}$ data line. This indicates that the phenomena responsible for the intensity dependence of the zero-crossing time is related to multiple excitation of RCs. It is also unambiguous from Fig. 9B that the zero-crossing time is specific to which pigment pool is excited. Not surprisingly, data at pump wavelengths of $675 \mathrm{~nm}$ (data not shown) give 

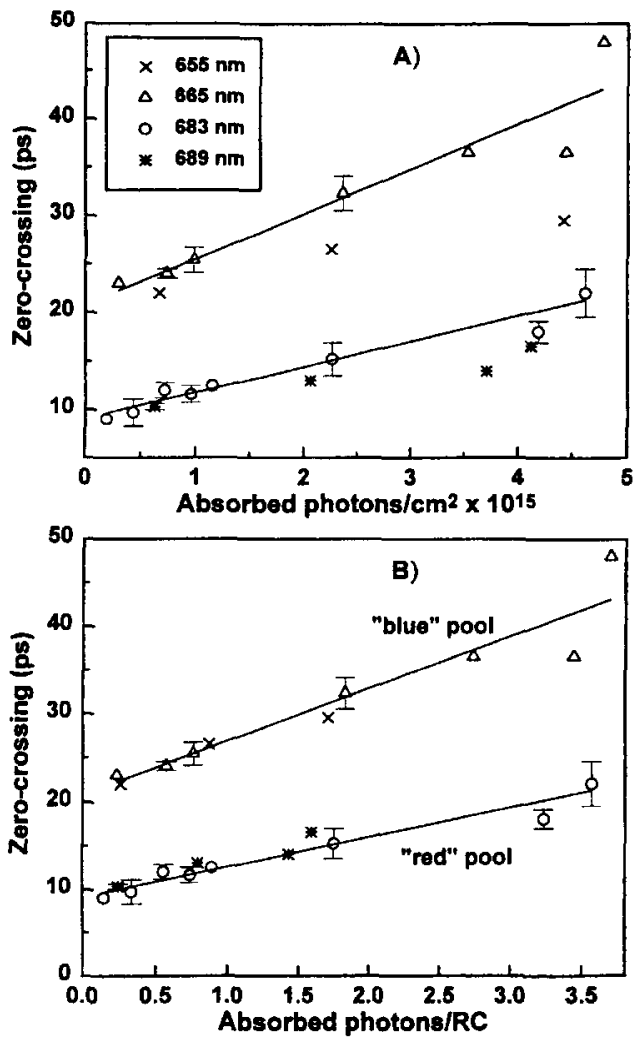

Fig. 9. Zero-crossing time (time after the laser flash at which the transient absorption signal crosses $\Delta A=0$; see Fig. 2) in isolated PSII RCs for a probe wavelength of $544 \mathrm{~nm}$. Lines in both (A) and (B) are linear fits to the 665 and $683 \mathrm{~nm}$ data for all pump energies. (A) As a function of absorbed photons per $\mathrm{cm}^{2}$. Note the lesser slope but similar intercept for $655 \mathrm{~nm}$ excitation as compared to $665 \mathrm{~nm}$ excitation, and similarly with 689 and $683 \mathrm{~nm}$ excitation wavelengths. (B) As a function of absorbed photons per RC, which shows effects of multiple excitation of individual RCs. All "blue" pool excitation data now lie on the same line, and all "red" pool data lie on a different line.

zero-crossing times in-between those for selective excitation of the two pools.

The longer zero-crossing time for the "blue" excitation is consistent with the data presented earlier in the paper. Since "blue" excitation results in a higher amplitude at $t=0$ than "red" excitation, it therefore would take longer to reach $\Delta A=0$ for "blue" excitation if the decay profiles were identical. As discussed above, all three decay constants are slightly slower for "blue" excitation than for "red", so this too contributes to a longer zero-crossing time for "blue" excitation.
The increase of the zero-crossing time with increasing pump energy is also consistent with the data presented earlier in the paper. In a completely linear regime, doubling the pump energy should be simply equivalent to multiplying the amplitude of the decay curve by a factor of two, which will of course leave the zero-crossing time unchanged. As shown earlier (see Figs. 6 and 8), increasing the pump energy into the regime where multiple excitation of individual $\mathrm{RCs}$ occurs results in complete saturation of the shelf, but the $t=0$ amplitude continues to increase, albeit at a lesser slope. Since neither the rates nor the relative amplitudes of the kinetic components change to any appreciable extent as a function of pump energy, the decay profile remains fundamentally unchanged. The net result of the same decay profile starting at a higher positive value but ending at the same negative value is that the point at which the decay crosses zero increases in time.

The zero-crossing time is also a measure of sample activity. As the sample degrades and the shelf rises ( $\Delta A$ more positive, indicating less charge separation), the zero-crossing is pushed out in time. Badly degraded sample do not even cross the baseline (data not shown). The zero-crossing times that we have measured with low pump energies $(\sim 10$ ps with 683 and $689 \mathrm{~nm}$ pump wavelengths) appear to be shorter than any previously found in the literature for direct excitation into the "red" pool (18-30 ps) $[28,30,32]$.

\subsection{Transient absorption spectra}

Transient absorption spectra were recorded at several time delays for both 665 and $683 \mathrm{~nm}$ excitation using $250 \mathrm{~nJ}$ pump pulses. The same sample was used for all spectra presented here, although other samples were measured and produced identical results. A weak but nonzero transient spectrum before $t=0$ (due to a small fraction of RCs in the triplet state from the previous pump pulse $1.5 \mathrm{~ms}$ earlier) was recorded and subtracted from the transient spectra at positive temporal delays. Fig. 10A shows transient spectra at a delay of 500 ps from 500 to $610 \mathrm{~nm}$. Within the signal to noise of the data, the spectra with 665 and $683 \mathrm{~nm}$ excitation wavelengths are identical over the entire region. Thus, after excitation energy transfer and charge separation are com- 

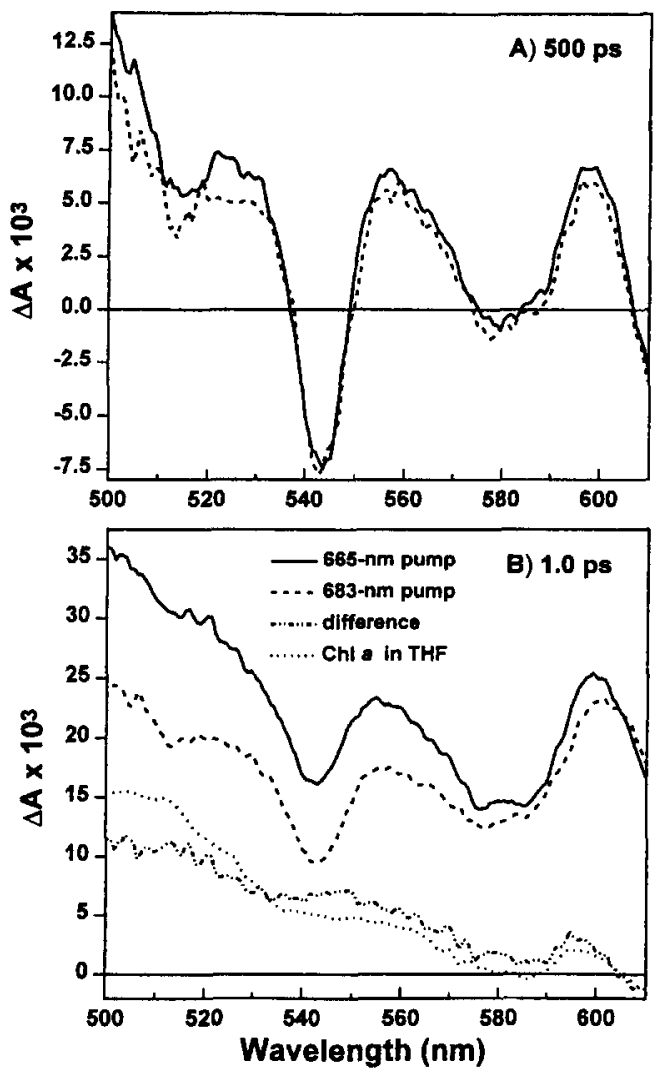

Fig. 10. (A) Transient absorption spectra at $500 \mathrm{ps}$ after the laser flash for pump wavelengths of 665 and $683 \mathrm{~nm}$ in isolated PSII RCs. The data, including the Pheo $a$ bleach region, are identical to within the $S / N$, indicating identical degrees of charge separation. (B) Transient absorption spectra at $1.0 \mathrm{ps}$ after the laser flash for pump wavelengths of 665 and $683 \mathrm{~nm}$. Also shown is the 665 minus $683 \mathrm{~nm}$ difference spectrum. Note the absence of any feature in the difference spectrum in the region of the Pheo $a$ bleach, indicating identical depopulation of the Pheo a ground state at this time for both pump wavelengths. The same PSII RC sample was used for all four spectra. Also shown is the transient absorption spectrum of Chl $a$ in THF, which is strikingly similar to the 665 minus $683 \mathrm{~nm}$ difference spectrum.

plete, the RCs are spectrally indistinguishable. This confirms the conclusion of equal quantum yields for charge separation reached earlier based solely on the data of the shelf value at $544 \mathrm{~nm}$ (see Fig. 8). The spectra clearly show that not only is the absolute value of long-time $\Delta A$ at $544 \mathrm{~nm}$ independent of pump wavelength, but the depth of the feature (with respect to the shoulders at either side) is independent as well. This is significant as it is really the depth of the Pheo $a$ bleach that is the measure of the popula- tion of $\mathrm{P} 60^{+}-\mathrm{Pheo} a^{-}$(and ${ }^{1 *}$ Pheo $a$ ), not the position of the peak relative to $\Delta A=0$.

The transient absorption spectra at 1.0 ps for 665 and $683 \mathrm{~nm}$ pump wavelengths are shown in Fig. 10B. At this time, the spectra are clearly quite different, and the 665 minus $683 \mathrm{~nm}$ difference spectrum is also shown (on the same scale). The general trend is for the difference in the transient absorption spectra to be greater at shorter probe wavelengths. The difference (at $544 \mathrm{~nm}$ at $350 \mathrm{fs}$ ) was seen earlier in Fig. 6. The absence of any feature in the difference spectrum in the region of the Pheo $a$ bleach signifies that the extent of depopulation of the Pheo $a$ ground state at $1.0 \mathrm{ps}$ is independent of excitation wavelength. A measurement of the depth of the bleach with respect to the red shoulder at 558 $\mathrm{nm}$ confirms that the depth is the same for the two excitation wavelengths. The depth of the bleach at $1.0 \mathrm{ps}$ is already $\sim 50 \%$ of the maximum depth (cf. Fig. 10A). The spectrum of the Pheo $a$ bleach region at $500 \mathrm{fs}$ (data not shown) is indistinguishable from that at $1.0 \mathrm{ps}$. Our data are in sharp contrast with that of Rech et al. [16], which show no significant difference in the $520-580 \mathrm{~nm}$ region as a function of "red" versus "blue" pigment pool excitation at 3 ps. We have also compared the spectra at both 8 and $50 \mathrm{ps}$, and the differences between 665 and $683 \mathrm{~nm}$ excitation are still present, albeit diminishing (recall at $500 \mathrm{ps}$, the spectra are indistinguishable). Also shown in Fig. 10B is the transient absorption spectrum of Chl $a$ in THF at 14 ps (the in vitro Chl $a$ spectra do not change on this time scale, so these data can be legitimately compared to the $1.0 \mathrm{ps}$ spectra of the PSII RCs). This spectrum has been rescaled and vertically shifted to match the PSII RC difference spectrum. The features of the PSII RC difference spectrum are very similar to those of in vitro $\mathrm{Chl} a$, indicating that the state from which excitation energy transfer takes place in the PSII RC is (not surprisingly) an excited state of Chl $a$.

\section{Discussion}

\section{I. Subpicosecond excitation energy transfer}

The large differential in amplitude near $t=0$ (e.g., at $\sim 350 \mathrm{fs}$ ) that is manifest in Fig. 6 at all pump energies for 665 and $683 \mathrm{~nm}$ pump wave- 
lengths probing at $544 \mathrm{~nm}$ is a new observation. The same phenomena exists over virtually the entire $500-610 \mathrm{~nm}$ wavelength region at $1.0 \mathrm{ps}$ (see Fig. 10B), and is still present in transient spectra taken at 50 ps. The transient absorption signal at any given wavelength is a combination of stimulated emission, ground state photobleaching, and absorption of the transient species. There is no stimulated emission in this wavelength region. The contribution due to photobleaching of Chl $a$ is fairly small because of its weak ground state absorption in this wavelength region. Fig. 10B clearly shows that the depth of the Pheo $a$ bleach is identical at 1.0 ps for the two pump wavelengths, so there can be little or no contribution of Pheo $a$ bleaching to the 665 minus $683 \mathrm{~nm}$ difference spectrum. Assuming that there is no subpicosecond electron transfer occurring (there is no evidence for it), the differential in the amplitudes for the two pump wavelengths must be due to differences in the excited state-excited state extinction coefficients of the two pools of excited chromophores. Interestingly, the difference spectrum looks very similar to the transient spectrum of ${ }^{1 *} \mathrm{Chl}$ $a$ in vitro, and probably represents the excited singlet state of a "blue"'-pool Chl $a$ from which the slow energy transfer process occurs (see below). Regardless of the exact nature of the cause of the differences in the transient absorption spectra, the important fact is that there is a difference, meaning that excitation energy equilibration at $1 \mathrm{ps}$ is far from complete, in contrast with the $100 \mathrm{fs}$ equilibration proposed in 1992 by Durrant et al. [15]. In 1994, Rech et al. proposed [16] that while the main pool of the "blue" pigments equilibrates on the $100 \mathrm{fs}$ timescale, energy transfer from a secondary pool to the main "blue" pool occurs on a much slower timescale (tens of picoseconds). While our data cannot rule out the existence of subpicosecond energy transfer, the magnitude of the differential between the two spectra at $1.0 \mathrm{ps}$ calls into question the extent of subpicosecond equilibration that could be consistent with our observation. Furthermore, it has been suggested by Schelvis and Aartsma [43] that the observations which Durrant et al. [15] have interpreted as being due to subpicosecond energy transfer between the "blue" and "red" pigment pools may actually be a manifestation of exciton scattering within the P680 dimer itself.

\subsection{Slow excitation energy transfer}

In order to estimate the time for excitation energy transfer from the "blue" pigment pool to the "red" pool, we have simulated the kinetics based on the average fit values given in Table 1. The $683 \mathrm{~nm}$ calculated curve was then subtracted from the 665 $\mathrm{nm}$ curve, with the resulting curve shown in Fig. 11. Since the difference between the decays for the two pump wavelengths is due to the absence of the slow energy transfer step for "red"'-pool excitation, the decay of the difference curve must be related to energy transfer. The vast majority $(89 \%)$ of the amplitude of the difference curve decays with a time constant of $\sim 29 \mathrm{ps}$, although a small fraction decays with a $>100$ ps time constant (see Fig. 11). While the actual energy transfer rate cannot be determined from this number without knowledge of the kinetic scheme for energy and electron transfer processes in the $\mathrm{RC}$, it does provide an estimate. This energy transfer process also manifests itself as the increase in the amplitude (both absolute and relative) of the intermediate component for $665 \mathrm{~nm}$ excitation as compared to $683 \mathrm{~nm}$ excitation, as seen in Fig. 4.

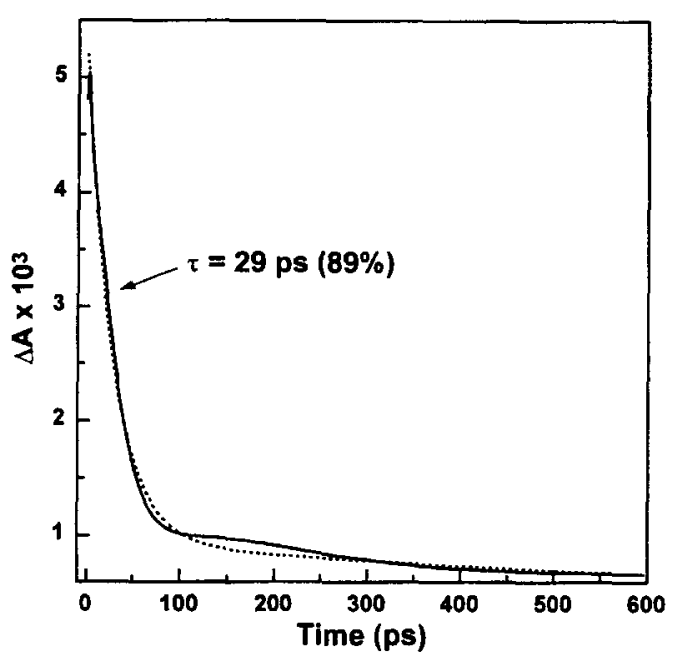

Fig. 11. Simulation of the excitation energy transfer kinetics. The fit parameters in Table 1 were used to calculate "average" decays at $544 \mathrm{~nm}$ for 665 and $683 \mathrm{~nm}$ pump wavelengths with $200 \mathrm{~nJ}$ pulses. The solid curve is the 665 minus $683 \mathrm{~nm}$ data. The dashed curve is a biexponential fit to the difference curve, with over $89 \%$ of the amplitude of the decay belonging to the $29 \mathrm{ps}$ component. 


\subsection{Intensity dependence}

Several ways of monitoring the effects of increasing pump intensity on the RC dynamics point to $\sim 250 \mathrm{~nJ}$ per pulse (for 665 and $683 \mathrm{~nm}$ ) being the maximum allowable energy for linearity for the spot size used in our experiments. We have both increased (into the nonlinear regime) and decreased (within the linear regime) the intensity by a factor of 4-5 from this point, without any apparent change in either the relative amplitudes or the time constants of the three components of the triple exponential fit to the data (see Figs. 4 and 5). We see no evidence of the appearance of an additional kinetic component that can be ascribed to annihilation phenomena, as has been seen for light harvesting complexes of PSII (LHC II) [44]. Since the isolated PSII RC has significantly fewer pigments than LHC II, it should be less susceptible to annihilation phenomena. Nevertheless, at the highest pump energies used in this study, a significant number of RCs should be multiply excited (see below), and one might expect annihilation to occur in these RCs. A possible explanation for the absence of any observed annihilation at high pump energies may be that it is occurring on a timescale that is difficult to observe given the triple exponential decay of the RCs. For the LHC II complexes, annihilation was easily discernible as a $\sim 25$ ps component appearing on a "natural" nanosecond long decay. Furthermore, (any) annihilation may not be occurring with a single rate constant, further complicating its detection. There has been recent evidence for the appearance of an additional kinetic component in isolated PSII RCs at higher excitation energies, but this observation is complicated by the use of an excitation wavelength of $400 \mathrm{~nm}$, which excites the high-energy Soret band of the Chl $a$ and Pheo $a$ molecules without any selectivity [33].

At $200 \mathrm{~nJ}$ per pulse with pump wavelengths of 665 and $683 \mathrm{~nm}$, we estimate a sample average of $\sim 0.7$ absorbed photons per RC, assuming linear absorption (the deviation from Beer's law at this pump level is estimated to be less than 15\%). To gain insight into the distribution of unexcited, singly excited, and multiply excited RCs, we have done a simple statistical simulation. We start with a sample of $n$ RCs and $0.7 n$ photons, all of which will be absorbed. All RCs start in the ground state (i.e., none are in the triplet state), which is a reasonable assumption given the $650 \mathrm{~Hz}$ repetition rate of our pump pulses and continuous stirring of the sample. Each photon is "absorbed" one-at-a-time, with the probability of absorption by a $\mathrm{RC}$ of a given excitation state being proportional to the number of $\mathrm{RCs}$ in that state. No attempt to take into account the effects due to sample thickness were made, so the simulation treats the system as an infinitely thin sample. With 0.7 absorbed photons per RC, we estimate $50 \%$ of the RCs are unexcited, with almost $70 \%$ of the excited RCs being singly excited.

\subsection{Charge separation}

It is instructive to consider the simplest behavior that could be anticipated from the RCs given a set of experiments that compare direct excitation of P680 at one pump wavelength with excitation of the accessory Chl $a$ at a different pump wavelength. Direct excitation of P680 would result in charge separation, and would be observed as a single exponential decay at probe wavelengths appropriate for $\mathrm{P} 680^{+}$and Pheo $a^{-}$. Excitation of the accessory Chl $a$ would result in the serial processes of excitation energy transfer to P680 followed by charge separation, and would be observed as a either a single or biexponential decay, depending on the relative rates of energy transfer and charge separation, and whether energy transfer is directly observable at the probe wavelengths. Obviously, neither case resembles the observed data. Even in the relatively straightforward case of (primarily) direct excitation into P680, a triple exponential decay ensues. The simplistic assignment of the relative degree of charge separation to each of the three kinetic components based on their relative amplitudes is probably not warranted, given the large shift to lower $\Delta A$ between 1 and 500 ps for the entire transient spectrum (in the 500-600 $\mathrm{nm}$ region, see Fig. 10), the exact origin of which is not clear.

Insight into the mechanism of primary charge separation in bacterial reaction centers has been gained from pre-reduction of the bacteriopheophytin primary electron acceptor with chemical reductants, such as sodium dithionite [45]. A similar strategy can be employed to reduce Pheo $a$ in the PSII RC prior to flash excitation $[2,18,46]$. Pre-reduction of Pheo $a$ to Pheo $a^{-}$prevents further photochemical reduction 
of this species. Under these conditions the transient absorption spectra and kinetics of the PSII RC should reflect only processes involving excited states of the remaining pigments, and possibly, earlier charge separated intermediates (if any exist) such as $\mathrm{P} 60^{+} \mathrm{Chl}$ $a^{-}$. Our results indicate that there is a delicate balance between the conditions needed to achieve high yield pre-reduction of Pheo $a$ and those necessary to avoid degradation of the protein. We have developed an experimental protocol which allows us to measure the transient absorption spectra and kinetics reproducibly in pre-reduced PSII RCs. Preliminary results using wavelength selective excitation of P680 indicate that both the amplitudes and time constants of each of the three kinetic components change in the "closed" PSII RC relative to those in the "open"' PSII RC [18]. It is quite likely that there is a great deal of "heterogeneity" in the protein environment of the pigments in the RC [21]. Given this and the close relationship between energy transfer and charge separation within the PSII RC, it is likely that all three kinetic components reflect the charge separation process in differing degrees. The data are complex and further experiments are in progress to ascertain whether the pre-reduction strategy will allow us to formulate a model for the charge separation process that accounts for all of the data.

\section{Conclusions}

Transient absorption kinetic data probing at 544 nm show that for both "red" and "blue" excitation of the composite $\mathrm{Q}_{y}$ band, the ensuing signal has a triple exponential decay. Excitation into the "blue" side of the band results in an increase in all three time constants, and an increase in the relative amplitude of the intermediate component. The difference decay for the two excitation wavelengths decays primarily with a $\sim 29$ ps time constant, and is attributed to excitation energy transfer. A very large amplitude difference at early time for the two excitation wavelengths is present, and calls into question the extent of $100 \mathrm{fs}$ energy transfer between the two pigment pools.

The pump energy intensity dependence shows two distinct types of sublinear behavior. The intensity dependence of the amplitude of the transient signal near $t=0$ is shown to be similar to that of Chl $a$ in vitro, and is ascribed to a simple deviation of Beer's law linear absorption due to a nontrivial population of excited chromophores. The intensity dependence of the shelf amplitude and the zero-crossing time are correlated with the number of absorbed photons per $\mathrm{RC}$, and are related to the limit of one charge separated ion pair per RC. Saturation effects appear at lower energies when pumping into the peak of the composite $\mathrm{Q}_{y}$ band, and at higher intensities when pumping further into the wings. While the highest energies result in the complete saturation of the Pheo $a$ bleach, there is no evidence for any kinetic changes that could be attributed to annihilation processes.

The zero-crossing time is shown to be an important indicator of the identity of the pigment pool being excited, the pump energy density, and sample activity, and its value can be determined without fitting the decay. Furthermore, unlike the absolute amplitudes at either $t=0$ or at long time (i.e., the shelf), which also do not require fitting to be established, the zero-crossing time is relatively independent of the mode matching of the pump and the probe beams, and as such is less susceptible to experimental artifacts.

The results of this work show that a careful examination of the excitation wavelength dependence of the kinetics of the Pheo $a$ bleach reveal kinetics sufficiently complex to make assignment of one of the three kinetic components to charge separation very difficult, if such an assignment is even possible. However, these results will serve as a foundation on which to base other, more sophisticated experiments such as liquid $\mathrm{He}$ temperature experiments (where excitation energy transfer is unidirectional), studies involving pre-reduction of Pheo $a$, and experiments that measure the actual rate of growth of the Pheo a bleach. Hopefully such experiments will enable the development of a model for energy and electron transfer within this protein that is consistent with the data that have already been obtained on this complex nanostructure for photochemical energy conversion.

\section{Acknowledgements}

This work was supported by the Divisions of Energy Biosciences and Chemical Sciences, Office 
of Basic Energy Sciences, US Department of Energy under contracts DE-AC36-83CH10093 (MS) and W-31-109-Eng-38 (MRW). G thanks the Department of Plant Biology, UIUC, Urbana, IL for support. SRG acknowledges an appointment to the Distinguished Postdoctoral Research Program sponsored by the US DOE Office of Science Education and Technical Information. The authors thank S. Toon for preparing the RCs used in this study, and Professor $\mathrm{R}$. Sension for making her manuscript available prior to publication.

\section{References}

[1] M. Seibert, in: The photosynthetic reaction center, Vol. 1, eds. J. Deisenhofer and J.R. Norris (Academic Press, San Diego, 1993) p. 319.

[2] O. Nanba and K. Satoh, Proc. Natl. Acad. Sci. USA 84 (1987) 109.

[3] K. Satoh, in: The photosynthetic reaction center, Vol. 1, eds. J. Deisenhofer and J.R. Norris (Academic Press, San Diego, 1993) p. 289.

[4] M. Seibert, R. Picorel, A.B. Rubin and J.S. Connolly, Plant Physiol. 87 (1988) 303.

[5] G. Döring, H.H. Stiehl and H.T. Witt, Z. Naturforsch. B 22B (1967) 639.

[6] R. Jankowiak, D. Tang, G.J. Small and M. Seibert, J. Phys. Chem. 93 (1989) 1649.

[7] H.-C. Chang, R. Jankowiak, N.R.S. Reddy, C.F. Yocum, R. Picorel, M. Seibert and G.J. Small, J. Phys. Chem. 98 (1994) 7725.

[8] S.L.S. Kwa, C. Eijckelhoff, R. van Grondelle and J.P. Dekker, J. Phys. Chem. 98 (1994) 7702.

[9] V.L. Tetenkin, B.A. Gulyaev, M. Seibert and A.B. Rubin, FEBS Letters 250 (1989) 459.

[10] J.R. Durrant, D.R. Klug, S.L.S. Kwa, R. van Grondelle, G. Porter and J.P. Dekker, Proc. Natl. Acad. Sci. USA 92 (1995) 4798.

[11] D. Tang, R. Jankowiak, M. Seibert, C.F. Yocum and G.J. Small, J. Phys. Chem. 94 (1990) 6519.

[12] M. Mimuro, T. Tomo, Y. Nishimura, I. Yamazaki and K. Satoh, Biochim. Biophys. Acta 1232 (1995) 81.

[13] P. Braun, B.M. Greenberg and A. Scherz, Biochemistry 29 (1990) 10376.

[14] M.-L. Groot, E.J.G. Peterman, P.J.M. van Kan, I.H.M. van Stokkum, J.P. Dekker and R. van Grondelle, Biophys. J. 67 (1994) 318.

[15] J.R. Durrant, G. Hastings, D.M. Joseph, J. Barber, G. Porter and D.R. Klug, Proc. Natl. Acad. Sci. USA 89 (1992) 11632.

[16] T. Rech, J.R. Durrant, D.M. Joseph, J. Barber, G. Porter and D.R. Klug, Biochemistry 33 (1994) 14768.
[17] J.P.M. Schelvis, P.I. van Noort, T.J. Aartsma and H.J. van Gorkom, Biochim. Biophys. Acta 1184 (1994) 242.

[18] S.R. Greenfield, M.R. Wasielewski, Govindjee and M. Seibert, in: Photosynthesis: from light to biosphere, Vol. 1, ed. P. Mathis (Kluwer, Dordrecht, 1995) p. 663.

[19] W. Zinth and W. Kaiser, in: The photosynthetic reaction center, Vol. 2, eds. J. Deisenhofer and J.R. Norris (Academic Press, San Diego, 1993) p. 71.

[20] V.A. Shuvalov, in: The photosynthetic reaction center, Vol. 2, eds. J. Deisenhofer and J.R. Norris (Academic Press, San Diego, 1993) p. 89.

[21] S.R. Greenfield and M.R. Wasielewski, Photosynth. Res. (1996), in press.

[22] T.A. Roelofs, M. Gilbert, V.A. Shuvalov and A.R. Holzwarth, Biochim. Biophys. Acta 1060 (1991) 237.

[23] A.R. Holzwarth, M.G. Müller, G. Gatzen, M. Hucke and K. Grievenow, J. Luminescence 60/61 (1994) 497

[24] A. Freiberg, K. Timpmann, A.A. Moskalenko and N.Y. Kuznetsova, Biochim. Biophys. Acta 1184 (1994) 45.

[25] M.R. Wasielewski, D.G. Johnson, M. Seibert and Govindjee, Proc. Natl. Acad. Sci. USA 86 (1989) 524.

[26] S.W. McCauley, A.P. Baronavski, J.K. Rice, M.L. Ghirardi and A.K. Mattoo, Chem. Phys. Letters 198 (1992) 437.

[27] J.R. Durrant, G. Hastings, Q. Hong, J. Barber, G. Porter and D.R. Klug, Chem. Phys. Letters 188 (1992) 54.

[28] G. Hastings, J.R. Durrant, J. Barber, G. Porter and D.R. Klug, Biochemistry 31 (1992) 7638

[29] J.R. Durrant, G. Hastings, D.M. Joseph, J. Barber, G. Porter and D.R. Klug, Biochemistry 32 (1993) 8259.

[30] L.B. Giorgi, J.R. Durrant, S. Alizadeh, P.J. Nixon, D.M. Joseph, T. Rech, J. Barber, G. Porter and D.R. Klug, Biochim. Biophys. Acta 1186 (1994) 247.

[31] G.P. Wiederrecht, M. Seibert, Govindjee and M.R. Wasielewski, Proc. Natl. Acad. Sci. USA 91 (1994) 8999.

[32] D.R. Klug, T. Rech, D.M. Joseph, J. Barber, J.R. Durrant and G. Porter, Chem. Phys. 194 (1995) 433.

[33] B. Donovan, L.A. Walker, II, C.F. Yocum and R.J. Sension, J. Phys. Chem. 100 (1996) 1945.

[34] H.M. Visser, M.-L. Groot, F. van Mourik, I.H.M. van Stokkum, J.P. Dekker and R. van Grondelle, J. Phys. Chem. 99 (1995) 15304.

[35] M.R. Wasielewski, D.G. Johnson, Govindjee, C. Preston and M. Seibert, Photosynth. Res. 22 (1989) 89.

[36] F. Vacha, D.M. Joseph, J.R. Durrant, A. Telfer, D.R. Klug, G. Porter and J. Barber, Proc. Natl. Acad. Sci. USA 92 (1995) 2929.

[37] S.R. Greenfield and M.R. Wasielewski, Opt. Letters 20 (1995) 1394.

[38] W.A. Svec, in: The Chlorophylls, ed. H. Scheer (CRC Press, Boca Raton, FL, 1991) p. 89.

[39] R.J. Porra, in: The chlorophylls, ed. H. Scheer (CRC Press, Boca Raton, FL, 1991) p. 38.

[40] R.V. Danielius, K. Satoh, P.J.M. van Kan, J.J. Plijter, A.M. Nuijs and H.J. van Gorkom, FEBS Letters 213 (1987) 241.

[41] Q. Hong, J. Durrant, G. Hastings, G. Porter and D.R. Klug, Chem. Phys. Letters 202 (1993) 183. 
[42] W. Demtröder, Laser spectroscopy (Springer, Berlin, 1982) p. 42.

[43] J.P.M. Schelvis and T.J. Aartsma, Chem. Phys. 194 (1995) 303.

[44] T. Bittner, K.-D. Irrgang, G. Renger and M.R. Wasielewski, J. Phys. Chem. 98 (1994) 11821.
[45] E.C. Kellogg, S. Kolaczkowski, M.R. Wasielewski and D.M. Tiede, Photosynth. Res. 22 (1989) 47.

[46] H.A. Frank, Ö. Hansson and P. Mathis, Photosynth. Res. 20 (1989) 279. 